\title{
Molecular modeling of low bandgap diblock co-oligomers with $\pi$-bridges for applications in photovoltaics
}

\author{
Gabriel G.B. Alves ${ }^{\mathrm{a}, \mathrm{b}}$, Eliezer F. Oliveira ${ }^{\mathrm{c}}$, Augusto Batagin-Neto ${ }^{\mathrm{d}, *}$, Francisco C. Lavarda ${ }^{\mathrm{b}}$

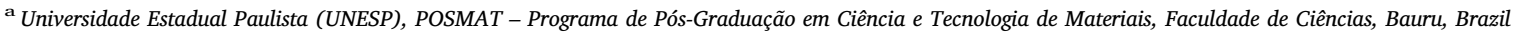 \\ ${ }^{\mathrm{b}}$ Universidade Estadual Paulista (UNESP), Faculdade de Ciências, Bauru, SP, Brazil \\ ${ }^{\mathrm{c}}$ Grupo de Sólidos Orgânicos e Novos Materiais (GSONM), Instituto de Física Gleb Wataghin (IFGW), Universidade Estadual de Campinas (UNICAMP), Campinas, SP, \\ Brazil \\ ${ }^{\mathrm{d}}$ Universidade Estadual Paulista (UNESP), Câmpus Experimental de Itapeva, Itapeva, SP, Brazil
}

\section{A R T I C L E I N F O}

\section{Keywords:}

Electronic structure calculation

Polythiophene

Polypyrrol

$\pi$-bridge

Oligomeric system

Photovoltaics

\begin{abstract}
A B S T R A C T
Recently, the diblock co-oligomers concept, a new design method to obtain conjugated organic compounds for varied applications in photovoltaics was proposed. These materials combines the interesting properties of extended systems and the versatility of small molecules, leading to low bandgap materials with improved properties, such as adjustable open circuit voltages and promising optical responses. Aiming to evaluate possible improved routes for the design of such materials, in this report we present a study on the effect of $\pi$-bridges incorporation on the properties of diblock co-oligomers. Six different $\pi$-bridges were inserted between polythiofene (Th) and polypyrrol (Py) oligomers with five units, following the structure $[\mathrm{Th}]_{5}-[\pi$-bridge $]$ - $[\mathrm{Py}]_{5}$. The geometry optimization and optical absorption studies were carried out in the density functional theory (DFT) and time dependent-DFT (TD-DFT) frameworks, respectively, using the B3LYP correlation-exchange functional and 6-31G(d,p) basis set. The results point out that compounds with improved opto-electronic properties can be obtained by an appropriated choice of the $\pi$-bridges. The possible improvements are associated with the higher delocalization of the $\pi$-systems on the molecules, reduction of the effective optical/electronic bandgaps, high optical transition probability between the new highest occupied and lowest unoccupied molecular levels (HOMO-LUMO), optimized charge transport properties and reduced exciton dissociation energies.
\end{abstract}

\section{Introduction}

Organic compounds with low bandgaps have been considered interesting materials for varied applications, from solar cells to near-IR photodetectors and ambipolar field effect transistors, mainly due to their unique opto-electronic properties, high versatility of synthesis and relative low cost [1-3].

In particular, a great variety of low bandgap organic materials has been applied in photovoltaics, specially in bulk-heterojunction (BHJ) organic solar cells (OSCs) [2]. Such devices are based on a blend of two or more organic materials with distinct electron affinities, where at least one of them is an optically active compound with significant absorption in the solar spectrum. Such materials act as donors or acceptors of free charge carriers that are generated after the dissociation of bounded electron-hole pairs (called excitons) coming from the material photoexcitation $[4,5]$. Considering all the mechanisms involved in these devices, materials with low bandgaps have presented good results, mainly due to their relevant photophysical properties that lead to several advantages [6,3]. Among them it is possible to highlight: (i) the better adjustments between the frontier molecular levels (involving the lowest unoccupied and the highest occupied molecular orbitals, LUMO and HOMO, respectively) with the electrodes and other components present in the devices, which assures higher open circuit voltages and efficient charge transfer processes; (ii) broader overall absorption spectra, that leads to improved photon-harvesting efficiencies and total current densities, and (iii) lower exciton binding energies, that facilitates the exciton dissociation and the generation of free charges in the devices [1,7-10]. In this context, the engineering of materials with appropriate bandgaps is a very active research area for the development of both, polymeric and small molecules based devices [11,12,6,13].

The design of low bandgap polymer-based systems is generally carried out via the co-polymerization of subunits with dissimilar donor-acceptor properties (DA concept), or through the stabilization of the quinoidal conformation of the conjugated structures $[14,15]$. On the other hand, the bandgap engineering of small molecules is a more challenging issue, mainly due the typical reduced conjugation of these

\footnotetext{
* Corresponding author.

E-mail address: abatagin@itapeva.unesp.br (A. Batagin-Neto).
} 
systems. Compounds of interest are usually obtained via the DA approach $[1,14,16-19]$, however the obtained bandgaps are still high for some applications, specially for OSCs $[1,20]$.

In this context, a recent study has pointed out that the use of diblock co-oligomers could be considered as a relevant alternative for the design of improved low bandgap materials for applications in photovoltaics [21]. Given their reduced extension, such materials present the typical advantages associated with organic small molecules, as ease of synthesis and higher purity $[1,10,22]$, at the same time that it allows the mitigation of relevant setbacks associated with morphological problems and low fill factors (FF) commonly associated with polymeric systems $[22,23]$.

Similarly to co-polymerization process, the bandgap engineering of such systems is based on the combination of oligomeric blocks with distinct frontier orbital levels. However, given the spatial separation between the employed blocks (and then between their frontier orbitals spatial distributions) a good balance between the oligomers extension is vital to favor optical transitions between these states. For example, it was shown that for a oligothiophene-oligopyrrole system the optimal blocks extension is around 5 units $\left([\mathrm{Th}]_{5}-[\mathrm{Py}]_{5}\right)[21]$.

An effective HOMO and LUMO distribution on the diblock structure is an important feature to obtain compounds with intermediate optoelectronic properties in relation to their constituting blocks. A common strategy in co-polymeric systems is the insertion of small molecules with strong $\pi$-systems between two distinct monomeric structures [24]. The main role of these compounds is establishing an effective connection between the $\pi$-systems of the co-polymer constituents and for this reason it is generically called as a $\pi$-bridge [25].

In the context of diblock co-oligomers, the presence of such bridges could indeed lead to significant improvements, not only in the $\pi$-system extension, but also in the resulting bandgaps. In this sense, here we evaluate the influence of $\pi$-bridges insertion on the opto-electronic properties of the $[\mathrm{Th}]_{5}-[\mathrm{Py}]_{5}$ diblock co-oligomer as a study case for the proposition of new materials with improved features for applications in OSCs. The obtained results indicate that an appropriate choice of the $\pi$-bridges can indeed lead to low bandgap materials $\left(E_{g} \sim 0.8 \mathrm{eV}\right)$ with interesting properties for photovoltaic applications, as higher HOMO/ LUMO overlap (with active optical transitions), lower exciton binding energies, and lower electron reorganization energies. In addition to the improvement of materials for photovoltaics, the obtained results also suggest an interesting design strategy for others varied applications employing distinct organic small molecules.

\section{Materials and methodology}

Fig. 1 illustrates the compounds evaluated in this study. The choice of thiophene and pyrrole structures with five units (pentamers) for the design of the diblock co-oligomers $\left([\mathrm{Th}]_{5}-[\mathrm{Py}]_{5}\right)$ was based on a previous work of our group, where it was demonstrated that such systems result in low bandgap materials with improved opto-electronic properties [21]. In particular, in that work it was shown that the diblock cooligomers properties are modulated by the resulting HOMO/LUMO overlap, mainly at the diblocks interface. In this context, aiming to improve this feature, six distinct $\pi$-bridges has been considered: benzo [1,2-c:4,5]bis[1,2,5]thiadiazole (BBT), benzothiadiazole (BT), carbazole (CAR), thiadiazoloquinoxaline (TDQ), thiophenopyrazine (TP), and vinyl (VIN). The choice of these bridges was based on both, spatial and energetic issues: (i) it was selected compounds with strong $\pi$-systems to facilitate the spatial distribution of $\pi$-electrons along the two oligomers; and (ii) the bridges should present specific energy level distributions in relation to the HOMO and LUMO of the unmodified diblock co-oligomer. In particular, regarding the last issue it was tested three distinct configurations: (a) bridges with LUMO levels inside the bandgap of the original diblock co-oligomer (typical acceptors), (b) bridges with HOMO levels inside the bandgap of the original diblock cooligomer, and (c) bridges with frontier levels outside the bandgap of the original system, aiming to investigate the effect of the formation of new intermediate states.

Preliminary dihedral analyses were performed for each system employing monomeric structures $\left([\mathrm{Th}]_{1}-[\pi\right.$-bridge $\left.]-[\mathrm{Py}]_{1}\right)$. For this purpose, four distinct conformations were constructed considering dihedrals of $0^{\circ}$ and $180^{\circ}$ between $[\mathrm{Th}]_{1}-[\pi$-bridge $]$ and $[\mathrm{Py}]_{1}-[\pi$-bridge $]$ subunits, which were then fully optimized. Since planar structures were obtained for all the monomer-based systems, restricted geometry optimizations of planar configurations have been carried out for $[\mathrm{Th}]_{5^{-}}$ $[\pi$-bridge $]-[\mathrm{Py}]_{5}$ structures. This approach is indeed relevant for the description of organic thin films, since the formation of planarized structures should be the expected after solvent evaporation [26].

All the geometry optimizations were performed in the framework of the density functional theory (DFT), with Becke's LYP (B3LYP) exchange-correlation functional [27-29] and 6-31G(d,p) basis set. In fact, there is a multitude of functionals available in the literature. However, in the case of oligomers and polymers it is known that their performance are quite dependent of the property under consideration, which suggests the necessity of employing distinct approaches to reproduce the structural, electrical and optical properties of these systems in a appropriate way [30-32,29,33-36]. Since in the present report we are more interested in evaluating tendencies than providing precise electronic parameters, the DFT/B3LYP approach have been chosen because, in average, it provides a reasonable description of both optical and electronic properties of non-extended oligomeric structures [29,34,36], being then appropriated to our systems.

The theoretical absorption spectra of the compounds were evaluated in a time dependent DFT approach (TD-DFT) [37], using the same

Diblock n-bridged co-oligomers<smiles>c1c[nH]c(-c2ccc(-c3ccc(-c4ccc(-c5ccc(-c6ccc(-c7ccc(-c8ccc(-c9ccc(-c%10ccc(-c%11cccs%11)s%10)s9)s8)[nH]7)s6)[nH]5)[nH]4)s3)[nH]2)c1</smiles>

\section{Bridges}

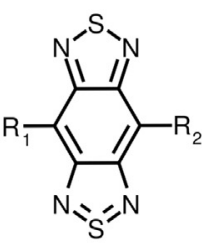

BBT
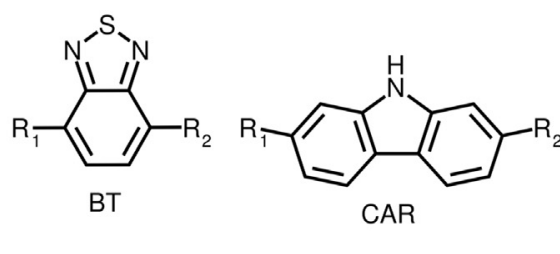

CAR

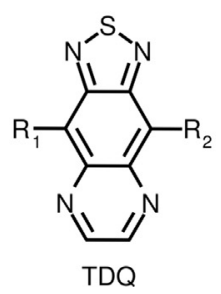<smiles>[R]N1C=CN([R])c2cscc21</smiles><smiles>[R]C=C[Y]</smiles>

Fig. 1. Schematic representation of the investigated systems and associated bridges. 


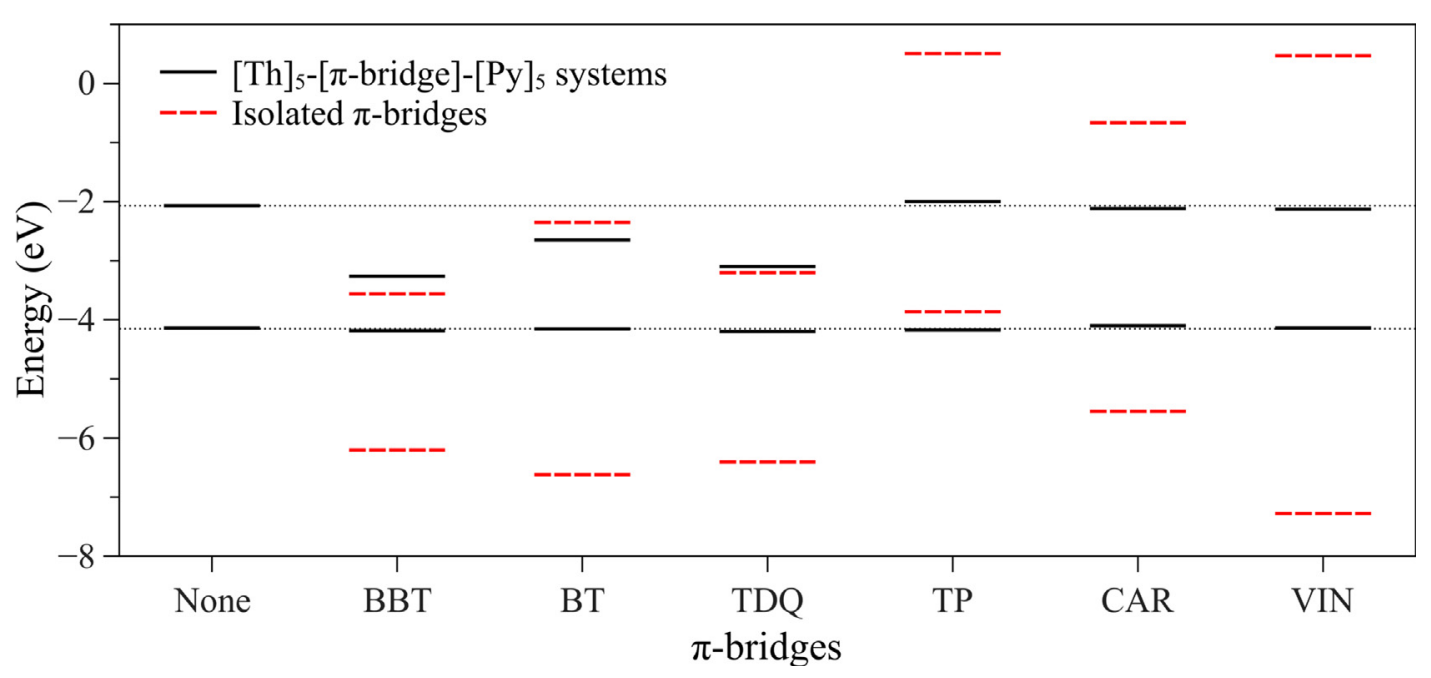

Fig. 2. Frontier molecular levels for the isolated bridges and resulting diblock compounds.

functional and basis set employed in the geometry optimizations. All the calculations were carried out with the aid of Gaussian 09 computational package [38].

Aiming to better evaluate the performance of the bridged diblock co-oligomers in solar cells some simple descriptors associated with charge transport and exciton dissociation were estimated: (i) electron and hole reorganization energies $\left(\lambda_{e}\right.$ and $\left.\lambda_{h}\right)$ [39,40] and (ii) exciton binding energies $\left(E_{B X}\right)[40]$.

The reorganization energy $(\lambda)$ represent the energy required for the geometric relaxation during charge transfer processes, being inversely proportional to the charge carriers mobilities [40-44]. These values can also indicate which is the dominant charge transport mechanism in the systems: in general $\lambda_{h}$ is lower than $\lambda_{e}$ in donor materials where greater mobility of holes is expected, while the inverse relation is observed for acceptors [45-47]. $E_{B X}$, in turn, indicates the energy that is required to dissociate a photogenerated exciton in the molecule, leading to the formation of free charges in the devices [48]. In this sense, small $E_{B X}$ values are associated with efficient exciton dissociation, which is a desired characteristic for solar cells.

Supposing a compound $M$ with $N$ electrons, the electron $\left(\lambda_{e}\right)$ and hole $\left(\lambda_{h}\right)$ reorganization energies are obtained via Eqs. (1) and (2):

$\lambda_{e}=\left[E_{T}\left(\nu_{N+1}, N\right)-E_{T}\left(\nu_{N}, N\right)\right]+\left[E_{T}\left(\nu_{N}, N+1\right)-E_{T}\left(\nu_{N+1}, N+1\right)\right]$

$\lambda_{h}=\left[E_{T}\left(\nu_{N-1}, N\right)-E_{T}\left(\nu_{N}, N\right)\right]+\left[E_{T}\left(\nu_{N}, N-1\right)-E_{T}\left(\nu_{N-1}, N-1\right)\right]$

where $E_{T}\left(\nu_{N+k}, N+j\right)$ represents the total energy obtained via a single point calculation of the species $M^{-j}$ (with $N+j$ electrons) for which the nuclear positions (represented by $v_{N+k}$ ) were previously obtained from the geometry optimization of the $M^{-k}$ specie (where $k$ and $j$ represents the changes in the electron number, being equal to $-1,0$ and +1 for cationic, neutral and anionic species, respectively). For example, the first parcel of $\lambda_{e}$ is obtained from the difference between the total energy of the charged specie $M^{-1}(j=+1$, i.e. with the addition of one electron in relation to $M$ ) for which the geometry was previously optimized in the neutral state $(k=0), E_{T}\left(\nu_{N+1}, N\right)$, and the total energy of the neutral specie $M(j=0)$ for which the geometry was pre-optimized in the neutral state $(k=0), E_{T}\left(\nu_{N}, N\right)$. In order to better describe the real potential curves involved in the charge transfer processes, fully optimized structures were considered for the estimation of reorganization energies. The geometry optimizations and single point calculations followed the same above presented DFT approach.

The exciton binding energy of each system was estimated via Eq. (3) $[49,50]$ :
$E_{B X}=\left[E_{L U M O}-E_{\text {HOMO }}\right]-E_{\text {optical }}$

where $E_{\text {НОМO }}$ and $E_{L U M O}$ represent the HOMO and LUMO energies, estimated from the Kohn-Sham orbital energies, while $E_{\text {optical }}$ represents the lowest-singlet excited energy coming from TD-DFT calculations.

\section{Results and discussion}

Fig. 2 presents the $E_{\text {HOMO }}$ and $E_{L U M O}$ levels of the unmodified [Th $]_{5^{-}}$ $[\mathrm{Py}]_{5}$ diblock, isolated $\pi$-bridges and the resulting $[\mathrm{Th}]_{5}-[\pi$-bridge $]-$ $[\mathrm{Py}]_{5}$ systems. Note that the employed bridges present distinct frontier levels distributions in relation to the bandgap of the unmodified diblocks: while the $E_{L U M O}$ of BBT, BT and TDQ and the $E_{\text {HOMO }}$ of TP are located inside the diblock bandgap, CAR and VIN bridges present their frontier levels outside of this range.

In general it is possible to observe that the bridged co-oligomers present different $E_{\mathrm{HOMO}}$ and $E_{L U M O}$ in relation to unmodified [Th $]_{5-}$ $[\mathrm{Py}]_{5}$. In particular, the most prominent changes are observed for BBT, $\mathrm{BT}$ and TDQ bridges, which suggests the relevance of the bridge LUMO level position for the design of low bandgap materials. Interestingly this trend is not observed when the HOMO of the bridge is inside of the diblock bandgap, as evidenced in TP-based system, for which no significant effects are observed. Finally, for CAR and VIN no effect can be noticed.

Despite of the above presented consideration, it is interesting to note that the new $E_{L U M O}$ observed in the bridged oligomers present small deviations in relation to the $E_{L U M O}$ of the isolated bridges. This effect is apparently associated with interactions between the orbitals of the bridges and the frontier molecular orbitals of the $[\mathrm{Th}]_{5}-[\mathrm{Py}]_{5}$ systems, being less expressive when the bridge LUMO level is located at the center of the diblock bandgap (see TDQ case in relation to BBT and BT).

In addition to the energetic distribution of the frontier levels, another relevant information to evaluate and predict the opto-electronic properties of the bridged systems is the spatial distribution of these molecular orbitals on the co-oligomer structures. In general a significant HOMO/LUMO overlap is desired to ensure an effective contribution of the new frontier orbitals to the optical and electrical response of the obtained materials. Fig. 3 shows the spatial distribution of the HOMO and LUMO on the compounds. The result obtained for the non-modified diblock co-oligomer is also presented for comparison.

As can be noticed, the presence of the BBT bridge leads to the most representative HOMO/LUMO overlap, followed by TDQ and BT. In 


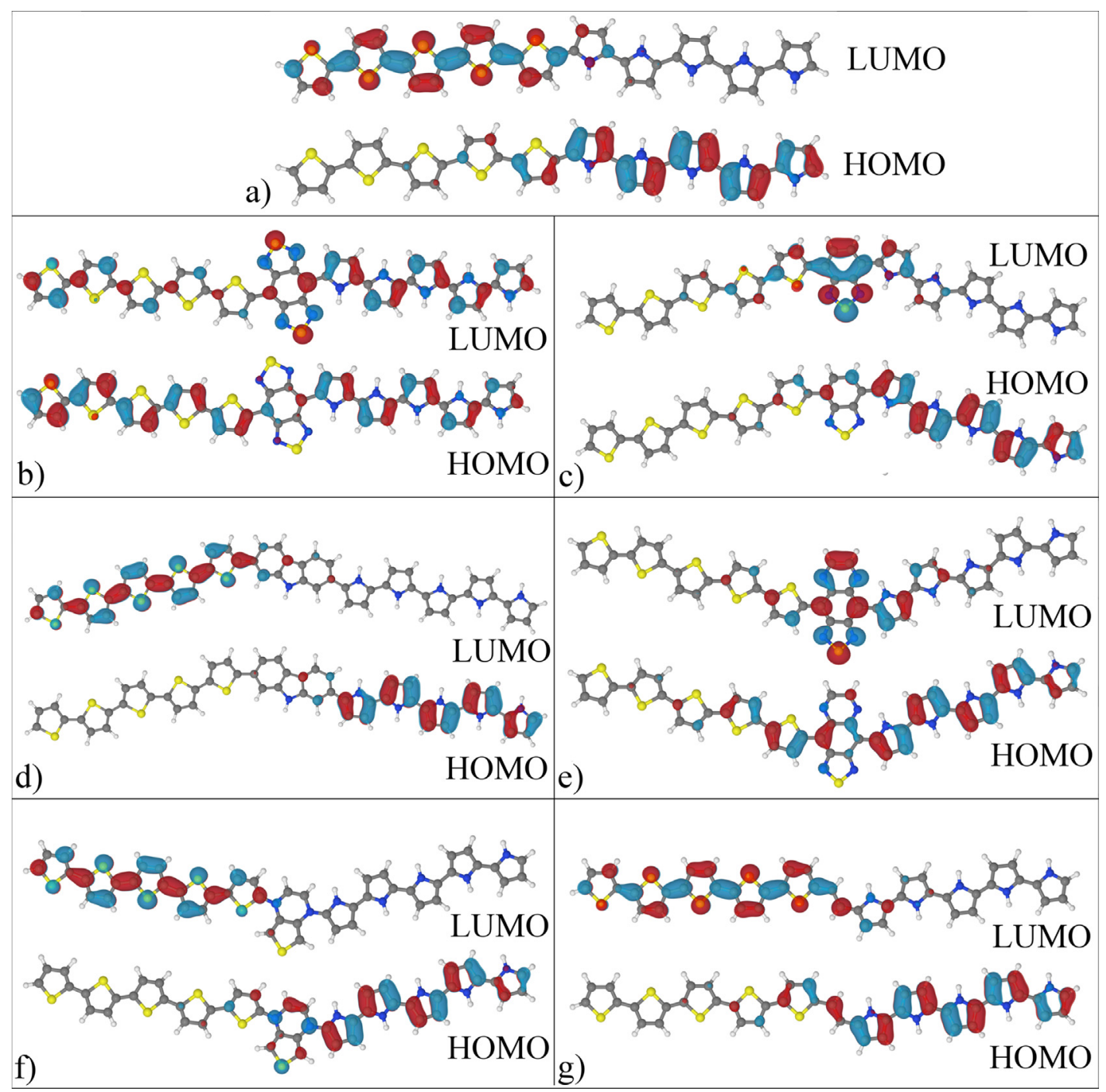

Fig. 3. HOMO and LUMO spatial distributions on the compounds structure: (a) $[\mathrm{Th}]_{5}-[\mathrm{Py}]_{5}$, (b) $[\mathrm{Th}]_{5}-\mathrm{BBT}-[\mathrm{Py}]_{5},(\mathrm{c})[\mathrm{Th}]_{5}-\mathrm{BT}-[\mathrm{Py}]_{5},(\mathrm{~d})[\mathrm{Th}]_{5}-\mathrm{CAR}-[\mathrm{Py}]_{5}$, (e) $[\mathrm{Th}]_{5}-$ TDQ- $[\mathrm{Py}]_{5}$, (f) $[\mathrm{Th}]_{5}-\mathrm{TP}-[\mathrm{Py}]_{5}$ and (g) $[\mathrm{Th}]_{5}-\mathrm{VIN}-[\mathrm{Py}]_{5}$.

particular, BBT promotes an optimized interconnection between the two oligomeric blocks, extending the electronic $\pi$-system along the entire compound structure. VIN presents intermediary results, that are similar to the non-modified diblock co-oligomer. Finally, CAR and TP lead to a reduction of the effective overlap in relation to unmodified diblock.

The above presented results indicate how relevant is the choice of the $\pi$-bridge for an effective orbital delocalization on the compound structure. Similar results have already been pointed out by Szarko and collaborators, in the study of oligomeric systems based on 3-hexyltiophene (3HT) oligomers and thienothiophene (TT) $\pi$-bridges [51]. In particular, Szarko et al. have shown that the presence of TT $\pi$-bridge between long 3HT blocks leads to the formation a localized state in the bandgap of the structures. For shorter oligomers, however, the new LUMO generated in the bandgap (associated with the bridge) is delocalized on the entire compound structure, leading to significant changes on the opto-electronic properties of the resulting systems.

From the work of Szarko it can be noticed that, when the frontier levels of the bridges and those of the unmodified compounds are close in energy, they can effectively interact, changing their overall spatial distribution. On the other hand, if the unmodified system is composed of extended oligomers, the interaction between the adjacent units of these structures is very strong and the effect of the $\pi$-bridges is quite small, leading to the generation of localized defect states.

Our results not only corroborate these observations but also allow the proposition of simple rules for the design of new low bandgap materials with effective optical responses. In particular for the BBT and TDQ based systems it is noticed that the LUMO of the bridges are located in the middle of the bandgap of the $[\mathrm{Th}]_{5}-[\mathrm{Py}]_{5}$, so they can effectively interact with both the frontier levels of the unmodified diblock. This interaction results in new HOMO and LUMO orbitals that present a significant spatial contribution of the bridge, extending the $\pi$-system on the diblock co-oligomers (see Fig. $2 b$ and e). On other hand, for BT based systems it is clear that the LUMO of the bridge interacts more effectively with the LUMO of the original diblock co-oligomer, changing its spatial distribution. However it is not able to interact with the diblock HOMO, which retains the same structure of the unmodified diblock co-oligomer, i.e. localized on the $\left[\mathrm{Py}_{5}\right]$ unit (see Fig. 2c).

Based on these results it is possible to conclude that very extended $\pi$-conjugated compounds can be obtained via the incorporation of specific $\pi$-bridges between small conjugated units. For this purpose, it is 


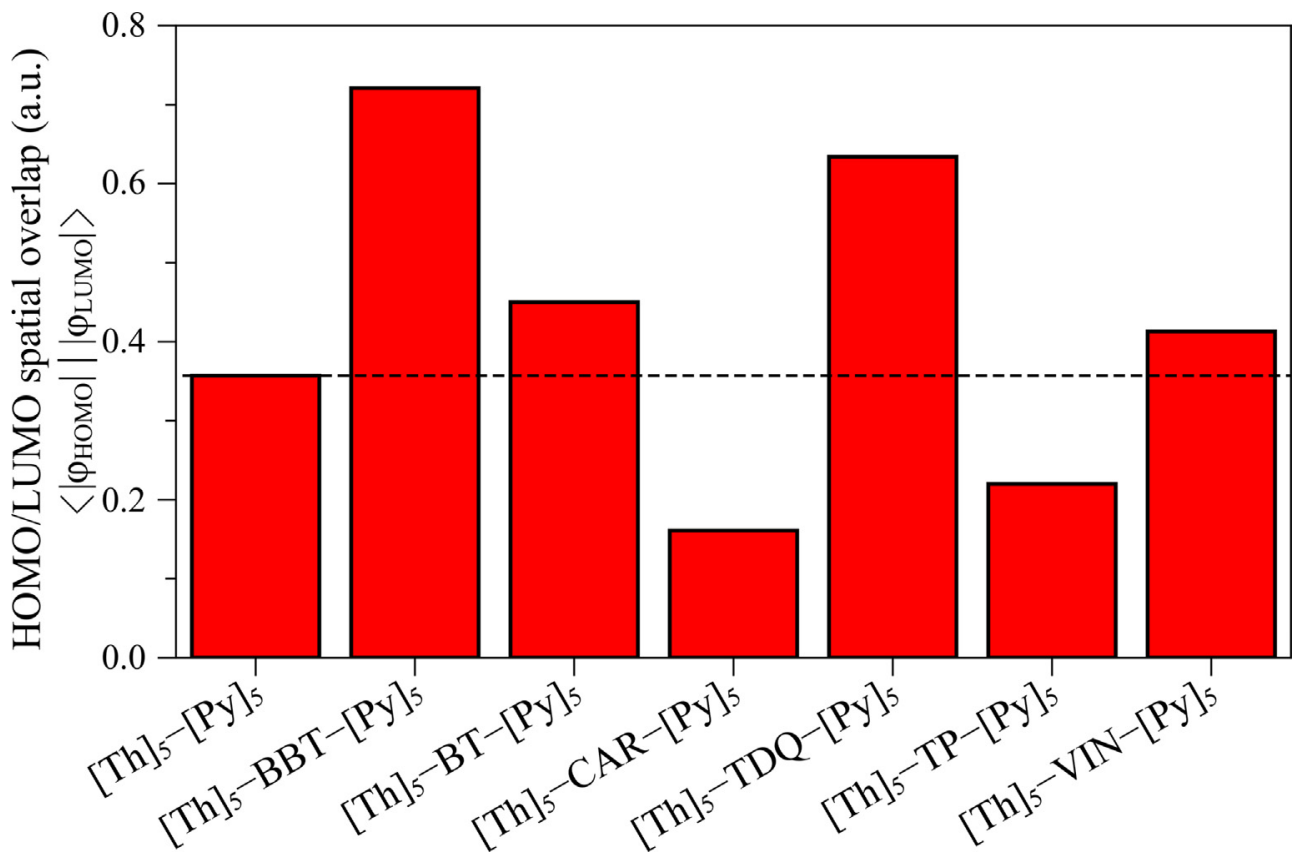

Fig. 4. HOMO/LUMO spatial overlap in $\pi$-bridged systems and unmodified $[\mathrm{Th}]_{5}-[\mathrm{Py}]_{5}$ structures.

necessary three requirements: (i) not so extended conjugated units; (ii) a bridge with a well formed $\pi$-system, and (iii) the LUMO of the bridge must be located inside the bandgap of the original diblock/dimeric system. It is important to highlight that these simple rules for the designing of low bandgap materials are not restricted to $\pi$-bridged diblock co-oligomers but they can be extended for other similar systems based on small molecules.

Fig. 4 presents a quantitative description of the compounds HOMO/ LUMO overlap, estimated via the spatial overlap matrix elements $\left\langle\left|\phi_{\text {HOMO }}\right||| \phi_{L U M O} \mid\right\rangle[52]$ that better illustrate the qualitative information presented in Fig. 3. Note that the frontier orbitals overlap are improved for BBT, TDQ, BT and VIN (in a decreasing order) and it is reduced for CAR and TP, as already discussed.

In order to better evaluate the effect of the $\pi$-bridges on the optical properties of the systems, the theoretical absorption spectra were evaluated. Fig. 5 shows the obtained results. The $[\mathrm{Th}]_{5}-[\mathrm{Py}]_{5}$ spectrum is also presented for comparisons (detailed information associated with the transitions are presented in the Supplementary Material).

It is worth to note how effectively BBT, TDQ and BT bridges influence the absorption spectra of the diblock co-oligomer, which is in accordance with the considerations presented above, i.e. a significant HOMO/LUMO overlap leads to more intense optical transitions

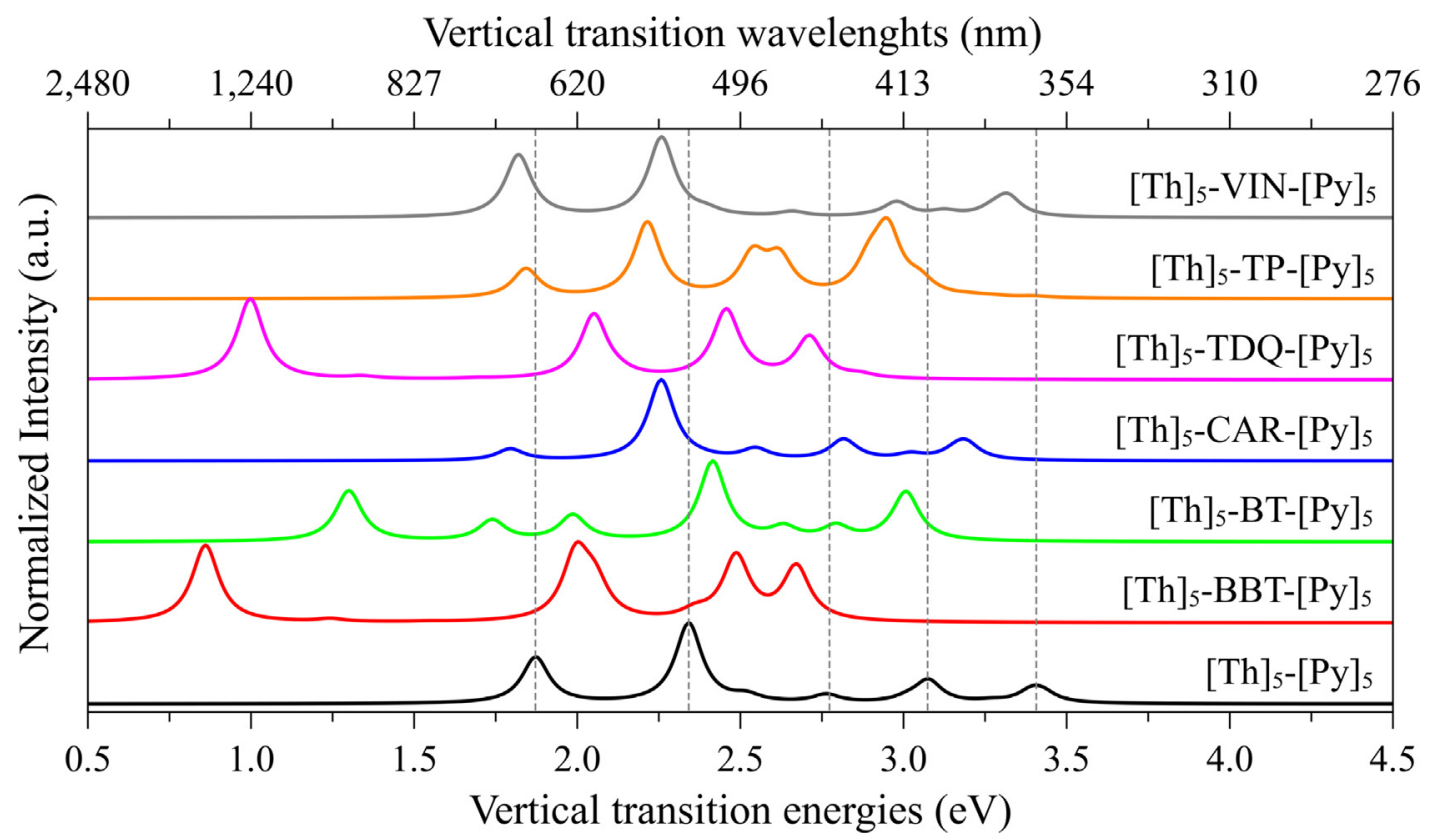

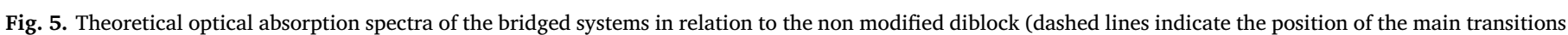
associated with $[\mathrm{Th}]_{5}-[\mathrm{Py}]_{5}$ ). 

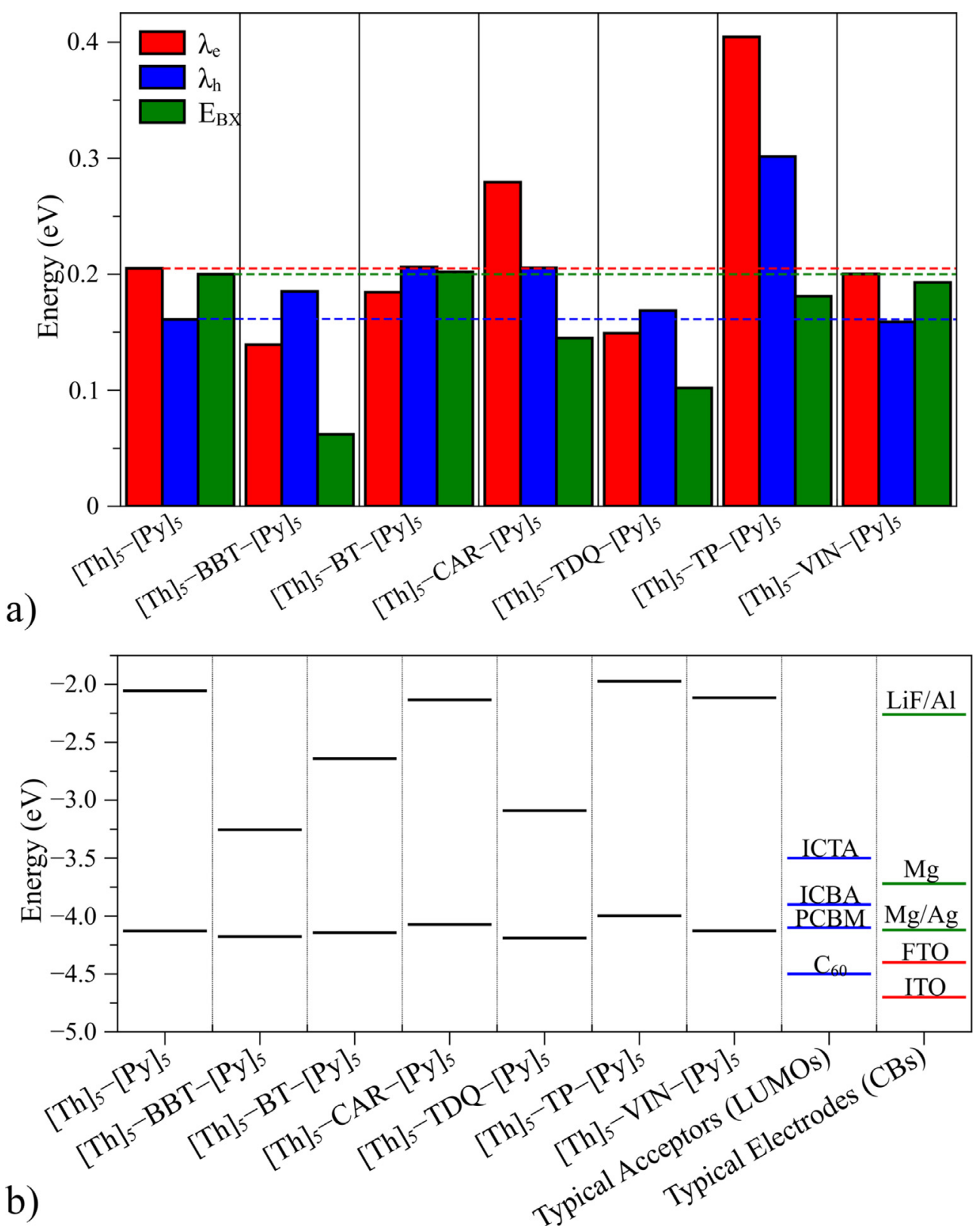

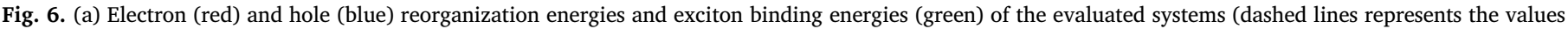

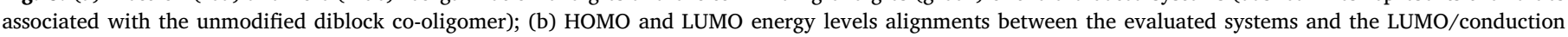

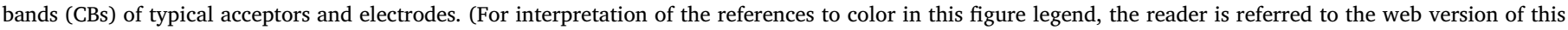
article.)

involving these orbitals (the lowest energy transitions). Note that the insertion of VIN and CAR bridges do not lead to significant changes on the main peak positions, as expected. The spectra of these compounds are quite similar to the original diblock one, with small shifts and changes on the relative amplitudes. Finally, the insertion of TP bridge does not lead to significant changes in the absorption at low energies, however some modifications are observed above $2.5 \mathrm{eV}$.

It is important to highlight how the information presented in Figs. 2 and 4 are linked to the resulting absorption spectra shown in Fig. 5. Fig. 2 shows that, when the LUMO of the bridge is located inside the original diblock co-oligomer gap, it presents a substantial contribution to the construction of the frontier orbitals of the bridged systems, which leads to a new material with a reduced bandgap. This feature, however, is not strong enough to ensure an effective optical response of these orbitals in the resulting systems, which can only be obtained if the transitions involving the new HOMO-LUMO levels are quite likely, which in a first approximation depends on their effective spatial overlap [53,54], as presented in Fig. 4.

According to these observations we can point out the relevance of the $\pi$-bridge to the transition intensities. The insertion of an appropriate $\pi$-bridge results in low energy optical transitions (HOMO $\rightarrow$ LUMO transitions) with the same amplitude (and sometimes more intense) than that observed in the unmodified diblock co-oligomer [21,51].

In order to evaluate the performance of the bridged systems in photovoltaic devices, some electronic descriptors associated with charge transfer, exciton dissociation and charge collection were evaluated. Fig. 6a presents the electron $\left(\lambda_{e}\right)$ and hole $\left(\lambda_{h}\right)$ reorganization energies, as well as the exciton binding energies $\left(E_{B X}\right)$ estimated for each bridged compound in relation to the unmodified $[\mathrm{Th}]_{5}-[\mathrm{Py}]_{5}$ 
diblock. Fig. 6b illustrates the frontier levels alignments of the obtained systems in relation to typical electron acceptor compounds [55] and electron and hole collection electrodes [56].

Note that BBT, TDQ and BT bridges lead to reduced $\lambda_{e}$ values (in a decreasing order) in comparison with the unmodified system. Higher values are observed for CAR and TP, while no significant changes are induced by the presence of VIN. Regarding hole transport, it is noticed that the presence of $\pi$-bridges in general leads to higher reorganization energies. However, for the most prominent bridges (BBT and TDQ) the change is just slightly higher than the unchanged diblock (lower than $0.03 \mathrm{eV}$ ). Finally, in relation to exciton binding energy it is noticed that BBT, TDQ and BT bridges lead to the most promising results, with a percentual reduction of $69.5,49.0$ and $27.5 \%$, respectively, in the $E_{B X}$ values. Less significant changes on this descriptor are observed for the other bridges.

Finally, through analysis of the levels alignments it is possible to observe that the recently proposed ICBA and ICTA fullerene derivatives can still act as effective acceptors for these low bandgap compounds. It is interesting to note that the use of PCBM is not appropriated for some systems and $\mathrm{C}_{60}$ is not indicated at all. Regarding the electrodes it is possible to note that some commonly employed materials can still be considered for these compounds, however, given the proximity of the frontier energy levels, they can play distinct effective roles. For instance, it is noticed that the efficiency of electron collection via $\mathrm{Mg}$ electrodes can be improved in the $\pi$-bridged systems at the same time that hole collection could also occur.

Based on the above presented results, among the systems investigated in this report, BBT and TDQ bridged systems have presented the most significant requirements for applications in solar cells. At the same time that such compounds show the lowest exciton binding energies and reorganization energies for electron transfer, the undesired effect associated with the increases of $\lambda_{h}$ is less expressive (lower than $2 k T$ at room temperature). In addition, these systems show an efficient HOMO/LUMO overlap, with active optical transitions, that points out them as effective low bandgap materials for varied applications. We consider that the present case study involving $[\mathrm{Th}]_{5}-[\mathrm{Py}]_{5}$ based systems indicates a relevant way to obtain new materials with relevant properties for photovoltaics that can pave the way for the design of new low bandgap materials.

\section{Conclusions}

The effect of the inclusion of distinct $\pi$-bridges in the diblock cooligomer structures of oligothiophene and oligopyrrole were investigated via electronic structure calculations.

Simple rules for the design of low bandgap materials based on the evaluation of frontier orbitals alignments between diblock/dimeric systems and isolated $\pi$-bridges have been proposed and discussed.

The results indicate that an appropriate choice of bridges can lead to materials with bandgaps as low as $0.8 \mathrm{eV}$ for the $[\mathrm{Th}]_{5}-[\pi \text {-bridge }]_{-}[\mathrm{Py}]_{5}$ compounds, which can be eventually lower for other systems. The effective HOMO/LUMO overlap leads to improved opto-eletronic and charge transport properties. In particular very promising results were obtained for BBT and TDQ bridges in relation to the original diblock cooligomer. These bridged systems present the lowest exciton binding energies and improved reorganization energies for electron transfer, as well as a substantial HOMO/LUMO overlap.

The results coming from this case study indicate an interesting route for the design of new and optimized low bandgap materials for optoelectronic applications which combines relevant properties of polymers and organic small molecules.

\section{Data availability}

Data will be made available on request.

\section{Acknowledgements}

We would like to thank the Brazilian agencies CNPq (proc. 448310/ 2014-7), CAPES (procs. 88881.068492/2014-01 and 23038.004680/ 2015-01), and FAPESP (procs. 2012/21983-0, 2014/20410-1, and 2016/18499-0). This research was also supported by resources supplied by the Center for Scientific Computing (NCC/GridUNESP) of the São Paulo State University (UNESP).

\section{Appendix A. Supplementary material}

See Supplementary Material for details regarding the frontier energy levels of the isolated $\pi$-bridges and bridged systems, as well as additional information of the most relevant optical transitions. Supplementary data associated with this article can be found, in the online version, at http://dx.doi.org/10.1016/j.commatsci.2018.05. 027.

\section{References}

[1] L. Dou, Y. Liu, Z. Hong, G. Li, Y. Yang, Low-bandgap near-IR conjugated polymers/ molecules for organic electronics, Chem. Rev. 115 (23) (2015) 12633-12665, http://dx.doi.org/10.1021/acs.chemrev.5b00165.

[2] C. Liu, K. Wang, X. Gong, A.J. Heeger, Low bandgap semiconducting polymers for polymeric photovoltaics, Chem. Soc. Rev. 45 (17) (2016) 4825-4846, http://dx.doi. org/10.1039/C5CS00650C.

[3] G. Li, W.-H. Chang, Y. Yang, Low-bandgap conjugated polymers enabling solutionprocessable tandem solar cells, Nat. Rev. Mater. 2 (8) (2017) 17043, http://dx.doi. org/10.1038/natrevmats.2017.43.

[4] S. Rafique, S.M. Abdullah, K. Sulaiman, M. Iwamoto, Fundamentals of bulk heterojunction organic solar cells: An overview of stability/degradation issues and strategies for improvement, Renew. Sustain. Energy Rev. 84 (2018) 43-53, http:// dx.doi.org/10.1016/j.rser.2017.12.008.

[5] H. Zhou, L. Yang, W. You, Rational design of high performance conjugated polymers for organic solar cells, Macromolecules 45 (2) (2012) 607-632, http://dx.doi. org/10.1021/ma201648t.

[6] A. Azazi, A. Mabrouk, K. Alimi, Theoretical investigation on the photophysical properties of low-band-gap copolymers for photovoltaic devices, Comput. Theoretical Chem. 978 (1-3) (2011) 7-15, http://dx.doi.org/10.1016/j.comptc. 2011.08.020.

[7] O.A. Abdulrazzaq, V. Saini, S. Bourdo, E. Dervishi, A.S. Biris, Organic solar cells: A review of materials, limitations, and possibilities for improvement, Part. Sci. Technol. 31 (5) (2013) 427-442, http://dx.doi.org/10.1080/02726351.2013. 769470.

[8] Y. Li, Molecular design of photovoltaic materials for polymer solar cells: Toward suitable electronic energy levels and broad absorption, Acc. Chem. Res. 45 (5) (2012) 723-733, http://dx.doi.org/10.1021/ar2002446.

[9] G. Li, R. Zhu, Y. Yang, Polymer solar cells, Nat. Photon. 6 (3) (2012) 153-161, http://dx.doi.org/10.1038/nphoton.2012.11.

[10] A. Mishra, P. Bäuerle, Small molecule organic semiconductors on the move: promises for future solar energy technology, Angew. Chem. Int. Ed. 51 (9) (2012) 2020-2067, http://dx.doi.org/10.1002/anie.201102326.

[11] E.F. Oliveira, F.C. Lavarda, Copolymers with similar comonomers: Tuning frontier orbital energies for application in organic solar cells, Polym. Eng. Sci. 56 (4) (2016) 479-487, http://dx.doi.org/10.1002/pen.24275.

[12] D. Yang, H. Sasabe, T. Sano, J. Kido, Low-band-gap small molecule for efficient organic solar cells with a low energy loss below $0.6 \mathrm{ev}$ and a high open-circuit voltage of over 0.9 v, ACS Energy Lett. 2 (9) (2017) 2021-2025, http://dx.doi.org/ 10.1021/acsenergylett.7b00608.

[13] Z.-G. Zhang, Y. Yang, J. Yao, L. Xue, S. Chen, X. Li, W. Morrison, C. Yang, Y. Li, Constructing a strongly absorbing low-bandgap polymer acceptor for high-performance all-polymer solar cells, Angew. Chem. 129 (43) (2017) 13688-13692, http://dx.doi.org/10.1002/ange.201707678.

[14] Y.-C. Hung, C.-Y. Chao, C.-A. Dai, W.-F. Su, S.-T. Lin, Band gap engineering via controlling donor-acceptor compositions in conjugated copolymers, J. Phys. Chem. B 117 (2) (2013) 690-696, http://dx.doi.org/10.1021/jp3090974.

[15] R.A. Krüger, T.J. Gordon, T.C. Sutherland, T. Baumgartner, Band-gap engineering of polythiophenes via dithienophosphole doping, J. Polym. Sci., Part A: Polym. Chem. 49 (5) (2011) 1201-1209, http://dx.doi.org/10.1002/pola.24538.

[16] X. Guo, L. Xiao, W. Tang, B. Liu, R. Cui, Y. Zou, Synthesis and characterization of a new solution-processable star-shaped small molecule based on 5,6-bis(n-octyloxy)2,1,3-benzoselenadiazole for organic solar cells, J. Mater. Sci. 48 (17) (2013) 5833-5839, http://dx.doi.org/10.1007/s10853-013-7376-8.

[17] L. Zhang, W. Shen, R. He, X. Tang, Y. Yang, M. Li, Density functional study on the effect of aromatic rings flanked by bithiophene of novel electron donors in smallmolecule organic solar cells, Mater. Chem. Phys. 175 (2016) 13-21, http://dx.doi. org/10.1016/j.matchemphys.2016.01.062.

[18] H. Qin, L. Li, F. Guo, S. Su, J. Peng, Y. Cao, X. Peng, Solution-processed bulk heterojunction solar cells based on a porphyrin small molecule with $7 \%$ power conversion efficiency, Energy Environ. Sci. 7 (4) (2014) 1397-1401, http://dx.doi.org/ 
10.1039/C3EE43761B.

[19] Y. Liu, C.-C. Chen, Z. Hong, J. Gao, Y.M. Yang, H. Zhou, L. Dou, G. Li, Y. Yang, Solution-processed small-molecule solar cells: breaking the $10 \%$ power conversion efficiency, Sci. Rep. 3 (2013) srep03356, http://dx.doi.org/10.1038/srep03356.

[20] M. Scharber, N. Sariciftci, Efficiency of bulk-heterojunction organic solar cells, Prog. Polym. Sci. 38 (12) (2013) 1929-1940, http://dx.doi.org/10.1016/j. progpolymsci.2013.05.001.

[21] E.F. Oliveira, F.C. Lavarda, Design of diblock co-oligomers as low bandgap small molecules for organic solar cells, Mol. Simul. 43 (18) (2017) 1496-1501, http://dx. doi.org/10.1080/08927022.2017.1321759.

[22] Q. Zhang, B. Kan, F. Liu, G. Long, X. Wan, X. Chen, Y. Zuo, W. Ni, H. Zhang, M. Li, Z. Hu, F. Huang, Y. Cao, Z. Liang, M. Zhang, T.P. Russell, Y. Chen, Small-molecule solar cells with efficiency over 9\%, Nat. Photon. 9 (1) (2014) 35-41, http://dx.doi. org/10.1038/nphoton.2014.269.

[23] B. Kan, M. Li, Q. Zhang, F. Liu, X. Wan, Y. Wang, W. Ni, G. Long, X. Yang, H. Feng, Y. Zuo, M. Zhang, F. Huang, Y. Cao, T.P. Russell, Y. Chen, A series of simple oligomer-like small molecules based on oligothiophenes for solution-processed solar cells with high efficiency, J. Am. Chem. Soc. 137 (11) (2015) 3886-3893, http://dx. doi.org/10.1021/jacs.5b00305.

[24] X. Wang, Y. Sun, S. Chen, X. Guo, M. Zhang, X. Li, Y. Li, H. Wang, Effects of $\pi$-conjugated bridges on photovoltaic properties of donor- $\pi$-acceptor conjugated copolymers, Macromolecules 45 (3) (2012) 1208-1216, http://dx.doi.org/10. 1021/ma202656b.

[25] T.C. Parker, D.G.D. Patel, K. Moudgil, S. Barlow, C. Risko, J.-L. Brédas, J.R. Reynolds, S.R. Marder, Heteroannulated acceptors based on benzothiadiazole, Mater. Horizons 2 (1) (2014) 22-36, http://dx.doi.org/10.1039/C4MH00102H.

[26] E.F. Oliveira, F.C. Lavarda, Structure of P3HT in the solid state, J. Polym. Sci., Part B: Polym. Phys. 51 (18) (2013) 1350-1354, http://dx.doi.org/10.1002/polb. 23338.

[27] A.D. Becke, Density-functional thermochemistry. III. The role of exact exchange, J. Chem. Phys. 98 (7) (1993) 5648-5652, http://dx.doi.org/10.1063/1.464913.

[28] A.D. Becke, A new mixing of hartreefock and local density-functional theories, J. Chem. Phys. 98 (2) (1993) 1372-1377, http://dx.doi.org/10.1063/1.464304.

[29] E.F. Oliveira, J.C. Roldao, B. Milián-Medina, F.C. Lavarda, J. Gierschner, Calculation of low bandgap homopolymers: Comparison of TD-DFT methods with experimental oligomer series, Chem. Phys. Lett. 645 (2016) 169-173, http://dx.doi. org/10.1016/j.cplett.2015.12.059.

[30] T. Körzdörfer, J.-L. Brédas, Organic electronic materials: recent advances in the DFT description of the ground and excited states using tuned range-separated hybrid functionals, Acc. Chem. Res. 47 (11) (2014) 3284-3291, http://dx.doi.org/10. 1021/ar500021t.

[31] J. Tirado-Rives, W.L. Jorgensen, Performance of B3lyp density functional methods for a large set of organic molecules, J. Chem. Theory Comput. 4 (2) (2008) 297-306, http://dx.doi.org/10.1021/ct700248k.

[32] S.S. Zade, N. Zamoshchik, M. Bendikov, From short conjugated oligomers to conjugated polymers. Lessons from studies on long conjugated oligomers, Acc. Chem. Res. 44 (1) (2011) 14-24, http://dx.doi.org/10.1021/ar1000555.

[33] E.F. Oliveira, J. Shi, F.C. Lavarda, L. Lüer, B. Milián-Medina, J. Gierschner, Excited state absorption spectra of dissolved and aggregated distyrylbenzene: A TD-DFT state and vibronic analysis, J. Chem. Phys. 147 (3) (2017) 034903, http://dx.doi. org/10.1063/1.4993216.

[34] M. Wykes, B. Milián-Medina, J. Gierschner, Computational engineering of low bandgap copolymers, Front. Chem. 1, http://dx.doi.org/10.3389/fchem.2013. 00035 .

[35] U. Salzner, Electronic structure of conducting organic polymers: insights from timedependent density functional theory: Electronic structure of conducting organic polymers, Wiley Interdisciplinary Rev.: Computat. Mol. Sci. 4 (6) (2014) 601-622, http://dx.doi.org/10.1002/wcms.1194.

[36] T.M. McCormick, C.R. Bridges, E.I. Carrera, P.M. DiCarmine, G.L. Gibson, J. Hollinger, L.M. Kozycz, D.S. Seferos, Conjugated polymers: evaluating DFT methods for more accurate orbital energy modeling, Macromolecules 46 (10) (2013) 3879-3886, http://dx.doi.org/10.1021/ma4005023.

[37] M.A. Marques, C.A. Ullrich, F. Nogueira, A. Rubio, K. Burke, E.K.U. Gross, R. Beig, W. Beiglböck, W. Domcke, B.-G. Englert, U. Frisch, P. Hänggi, G. Hasinger, K. Hepp, W. Hillebrandt, D. Imboden, R.L. Jaffe, R. Lipowsky, H.v.Löhneysen, I. Ojima, D. Sornette, S. Theisen, W. Weise, J. Wess, J. Zittartz (Eds.), Time-Dependent Density
Functional Theory, Vol. 706 of Lecture Notes in Physics, Springer Berlin Heidelberg, Berlin, Heidelberg, 2006, http://dx.doi.org/10.1007/b11767107.

[38] M.J. Frisch, G.W. Trucks, H.B Schlegel, G.E Scuseria, M. Robb, J. Cheeseman, G. Scalmani, V. Barone, B. Mennucci, G.A.H. Petersson, H. Nakatsuji, M. Caricato, X. Li, H.P Hratchian, A.F Izmaylov, J. Bloino, G. Zheng, J.L Sonnenberg, M. Hada, D. Fox, Gaussian 09 (Revision A02), Gaussian Inc. Wallingford CT, 2009.

[39] E.F. Oliveira, F.C. Lavarda, Reorganization energy for hole and electron transfer of poly(3-hexylthiophene) derivatives, Polymer 99 (2016) 105-111, http://dx.doi. org/10.1016/j.polymer.2016.07.003.

[40] G.R. Hutchison, M.A. Ratner, T.J. Marks, Hopping transport in conductive heterocyclic oligomers: Reorganization energies and substituent effects, J. Am. Chem. Soc. 127 (7) (2005) 2339-2350, http://dx.doi.org/10.1021/ja0461421.

[41] R.A. Marcus, Chemical and electrochemical electron-transfer theory, Annu. Rev. Phys. Chem. 15 (1) (1964) 155-196, http://dx.doi.org/10.1146/annurev.pc.15. 100164.001103.

[42] H.M. McConnell, Intramolecular charge transfer in aromatic free radicals, J. Chem Phys. 35 (2) (1961) 508-515, http://dx.doi.org/10.1063/1.1731961.

[43] R.A. Marcus, Electron transfer reactions in chemistry. theory and experiment, Rev. Mod. Phys. 65 (3) (1993) 599-610, http://dx.doi.org/10.1103/RevModPhys.65. 599.

[44] M.D. Newton, N. Sutin, Electron transfer reactions in condensed phases, Annu. Rev. Phys. Chem. 35 (1) (1984) 437-480, http://dx.doi.org/10.1146/annurev.pc.35. 100184.002253.

[45] G. Garcia-Belmonte, A. Munar, E.M. Barea, J. Bisquert, I. Ugarte, R. Pacios, Charge carrier mobility and lifetime of organic bulk heterojunctions analyzed by impedance spectroscopy, Org. Electron. 9 (5) (2008) 847-851, http://dx.doi.org/10 1016/j.orgel.2008.06.007.

[46] V. Mihailetchi, H. Xie, B. deBoer, L. Koster, P. Blom, Charge transport and photocurrent generation in poly(3-hexylthiophene): Methanofullerene bulk-heterojunction solar cells, Adv. Funct. Mater. 16 (5) (2006) 699-708, http://dx.doi.org/10. 1002/adfm.200500420.

[47] C. Brückner, B. Engels, A theoretical description of charge reorganization energies in molecular organic p-type semiconductors, J. Comput. Chem. 37 (15) (2016) 1335-1344, http://dx.doi.org/10.1002/jcc.24325.

[48] C. Zhao, H. Ge, L. Jin, Q. Zhang, Z. Wang, W. Wang, S. Yin, Theoretical investigation on exciton-dissociation and charge-recombination processes of PC61bmPTDPPSe interface, J. Mol. Model. 22 (10) (2016) 241, http://dx.doi.org/10.1007/ s00894-016-3117-7.

[49] R.C. Hiorns, P. Iratabal, D. Bégué, A. Khoukh, R. De Bettignies, J. Leroy, M. Firon, C. Sentein, H. Martinez, H. Preud'homme, C. Dagron-Lartigau, Alternatively linking fullerene and conjugated polymers, J. Polym. Sci., Part A: Polym. Chem. 47 (9) (2009) 2304-2317, http://dx.doi.org/10.1002/pola.23311.

[50] L. Zhu, Y. Yi, L. Chen, Z. Shuai, Exciton binding energy of electronic polymers: A first principles study, J. Theoretical Comput. Chem. 07 (4) (2008) 517-530, http:// dx.doi.org/10.1142/S0219633608003939.

[51] J.M. Szarko, J. Guo, B.S. Rolczynski, L.X. Chen, Nanoscale structure, dynamics and power conversion efficiency correlations in small molecule and oligomer-based photovoltaic devices, Nano Rev. 2 (1) (2011) 7249, http://dx.doi.org/10.3402/ nano.v2i0.7249.

[52] M.J.G. Peach, P. Benfield, T. Helgaker, D.J. Tozer, Excitation energies in density functional theory: An evaluation and a diagnostic test, J. Chem. Phys. 128 (4) (2008) 044118, http://dx.doi.org/10.1063/1.2831900.

[53] M. Fox, Optical properties of solids, 2nd Edition, no. v. 3 in Oxford Master Series in Condensed Matter Physics, Oxford University Press, Oxford; New York, 2010.

[54] C.J. Cramer, Essentials of Computational Chemistry: Theories and Models, 2nd ed. Wiley, Chichester, West Sussex, England; Hoboken, NJ, 2004.

[55] M. Hu, C. Bi, Y. Yuan, Z. Xiao, Q. Dong, Y. Shao, J. Huang, Distinct exciton dissociation behavior of organolead trihalide perovskite and excitonic semiconductors studied in the same system, Small 11 (18) (2015) 2164-2169, http://dx.doi.org/10 1002/smll.201402905.

[56] L. Ma, Organic light-emitting diode with enhanced efficiency, Patent: Internacional Classification H01L51/50, H01L51/00; Cooperative Classification H01L51/5072, H01L51/5056, H01L51/504, H01L51/5016, H01L51/0085, H01L51/0059, H01L51/0037, H01L51/5036, H01L51/0094, H01L2251/552, H01L51/0062, H01L51/0067; European Classification H01L51/50E3, H01L51/50E8, 2011. 